\title{
Comparative Evaluation of Dry Eye Following Cataract Surgery: A Study from North India
}

\author{
Dr. Mohana Sinha ${ }^{1}$, Dr. Abhik Sinha ${ }^{2}$, Dr. Bithi Chowdhury ${ }^{3}$ \\ IMBBS,DNB (Ophthalmology) Senior Resident, Ophthalmology Calcutta National Medical College, Kolkata \\ 2MBBS, MD(Community Medicine), PGDGM Assistant Professor, Community Medicine, R.G Kar Medical \\ College, Kolkata \\ 3MS(Ophthalmology), FRCS Senior Eye Specialist Hindu Rao Hospital, New Delhi
}

\begin{abstract}
Background: After cataract surgery many patients complain of foreign body sensation, irritation, redness, blurring of vision which are considered as unwanted effects of the surgery. Aim: To compare dry eye disease following SICS and Phacoemulsification. Material and methods: The present study is a prospective, randomized study conducted on sixty nine patients, from July 2009 to June 2011, attending Outpatient Department of Department of Ophthalmology in a municipal hospital of Delhi. Complete ocular surface examination was done which included questionnaire using OSDI score and slit lamp bio microscope examination, tear film break up time, corneal fluorescein staining, tear meniscus height, Schirmer test - I and Impression cytology. Data analysis was done using SPSS version 16. Results: All the dry eye tests conducted on the eyes undergoing cataract surgery showed deterioration following surgery. Both SICS and Phaco surgery caused significant decrease in TMH, TBUT and ST-I values at 1 week, 1 month and 3 months. Fluorescein staining, OSDI score and impression cytology also showed deterioration in the follow up period. Conclusion: Both SICS and Phaco surgery can cause or aggravate dry eye and affect the dry eye test values in the post operative period upto 3 months.
\end{abstract}

\section{Main Text}

Dry eye disease is an ocular surface disorder which produces discomfort and reduced vision due to tear film instability. Dry eye per se is not a disease entity, but a symptom complex occurring as sequelae to deficiency or abnormalities of tear film, exposing the corneal and conjunctival epithelium to evaporation. Dry eye has become one of the most important factors influencing quality of Life (QOL) in elderly patients. Incidence of dry eye in the US has been estimated to be around 2.7 per year. Incidence of dry eye in India amongst eye out patient department patients has been estimated to be around $0.46 \%$ with a male: female ratio of $1: 1.22^{1}$

Cataract is the leading cause of blindness in the world. At the same time cataract surgery is the most successful and rewarding surgery in the field of ophthalmology. However, after cataract surgery many patients complain of foreign body sensation, irritation redness, blurring of vision which are considered as unwanted effects of the surgery (Cho,et al., 2009) ${ }^{2}$.These effects are worse in the elderly population and those with ocular surface disorder. These persist in some patients until they are managed by subsequent effective treatment ( $\mathrm{Li}$, et al., 2007) ${ }^{3}$. Some studies have reported aggravation of dry eye symptoms and signs after cataract surgery (Ram, et al., 1998 and 2002) ${ }^{4}$ and 5 . Thus inspite of a perfect cataract surgery and a good snellen visual acuity the patients may remain dissatisfied.

In conventional cataract surgery a large incision is made at the limbus denervating the superior half of the cornea leading to corneal desensitization with subsequent complications. In the post operative period prolonged use of antibiotic and steroid drops along with the presence of sutures further aggravates the dry eye condition. In phacoemulsification the size of the incision is smaller, surgical time is reduced and there is milder postoperative inflammation ${ }^{6}$. However little is known of the effect of the effect of small incision cataract surgery on dry eye status using phacoemulsification or manual SICS.

We studied the prevalence of dry eye symptoms in patients with questionnaires, clinical examination and impression cytology.

It is our endeavour in this study to evaluate the clinical course of dry eye following cataract surgery. There is no Indian study reported in literature where impression cytology has been used to investigate and diagnose dry eye disorder following cataract surgery, to the best of our knowledge. In the present study our aim was to evaluate the clinical course of dry eye disease following cataract surgery by comparative analysis of dry eye parameters between the two surgical groups. 


\section{Materials and methods}

The study was a prospective, randomized study conducted in the Department of Ophthalmology from July 2009 to June 2011 on patients attending Outpatient Department (OPD) of Department of Ophthalmology, in a municipal hospital of Delhi.

Following were the inclusion and exclusion criteria:

Inclusion criteria:

1. Age $>50$ years.

2. Patients having unilateral or bilateral age related cataract with or without dry eye symptoms.

Exclusion criteria:

1. Age $<50$ years.

2. Pseudophakia in the other eye.

3. Cataract caused by an etiology other than age, e.g., trauma, uveitis, drug induced.

4. Pre-existing ocular diseases glaucoma, disorders of lids, conjunctiva, cornea, and sclera.

5. Chemical burns, radiation.

6. Use of contact lens.

7. Patients on chronic ocular medications.

8. Patients who have undergone corneal refractive surgery.

9. Patients who had ocular allergies, pterygia, or blepharitis.

The study was done on 69 consecutive patients attending the outpatient department, having cataract and were scheduled for cataract operation fulfilling the inclusion and exclusion criteria of the study. The patients were randomized into 2 groups:

Group (A) of 30 patients was scheduled for manual Small Incision cataract surgery.

Group (B) of 39 patients was scheduled for phacoemulsification.

The other unoperated eye of all cases served as control in both the groups.

For evaluating the effect of dry eye on Quality Of Life (QOL) items, Ocular Surface Disease Index (OSDI) score (Data on file, Allergan, Inc) ${ }^{7}$ was used. QOL items such as foreign body sensation, asthenopia, difficulty in accomplishing activities of daily life, and response to environmental conditions such as wind, was graded on a 4 grade scale.

\section{FOLLOW UP:}

The patients in the two groups were followed up at 1 week, 1 month and 3 months post-operatively by means of questionnaire (Ocular Surface Disease Index), and the ocular examinations related to dry eye disease. The examinations conducted were Tear film Breakup Time, fluorescein staining grading, Tear Meniscus Height, Schirmer's test-I and conjunctival impression cytology. Conjunctival impression cytology was done preoperatively and at 3 months follow-up visit. A standardized follow up proforma was used for follow up.

\section{ANALYSIS:}

The data collected was entered into an Excel spreadsheet and analyzed using statistical software (SPSS 16.0). The significance ( $\mathrm{p}$ value) was set at 5\%. Relevant statistical tests e.g., paired $\mathrm{t}$ test were applied when required for data interpretation.

\section{Observations and results}

Sixty nine eyes of 69 patients attending the outpatient department of Hindu Rao Hospital, Delhi were included in this study to investigate dry eye disease and analyse the pathogenic factors following cataract surgery from July 2009 to June 2011. The fellow eyes of the 69 patients were taken as control and were subjected to all the tests. The patients were randomized into 2 groups:

Group (A) of 30 patients was scheduled for manual Small Incision cataract surgery.

Group (B) of 39 patients was scheduled for phacoemulsification.

The patients in the two groups were followed up at 1 week, 1 month and 3 months post-operatively. Patients were also evaluated for all the possible pathogenic factors related to dry eye following phacoemulsification and manual small incision cataract surgery.

The observation and results were discussed by comparative analysis of dry eye parameters between the two surgical groups. 


\section{Comparative analysis of dry eye parameters between the two surgical groups:}

When we analysed the patients according to those who had undergone manual small incision cataract surgery and those who had undergone phacoemulsification, we found out that pre-operatively the mean and standard deviation of tear meniscus height, schirmer's test, tear film breakup time, ocular surface disease index and impression cytology were respectively $0.55 \pm 0.166,9.23 \pm 2.112,9.48 \pm 3.212,56.18 \pm 10.926$ and $208.80 \pm 59.120$ in patients who underwent SICS and $0.55 \pm 0.170,9.231 \pm 2.117,9.487 \pm 2.489,54.482 \pm 9.001$ and $237.937 \pm 238.1$ in patients who underwent Phacoemulsification.

Postoperatively, the mean and standard deviation for Ocular Surface Disease Index (OSDI) at 1 week was found out to be $50.45 \pm 10.247$ in SICS patients, $39.86 \pm 9.215$ at 1 month, $37.76 \pm 9.607$ at 3 months. In Phaco patients OSDI at week was $47.360 \pm 7.525$, at 1 month was $40.628 \pm 8.907$ and at 3 months was $38.051 \pm 8.428$.

We also found out the mean and standard deviation of tear film breakup time in SICS patients, which came out to be $7.57 \pm 2.285$ at 1 week, $6.80 \pm 2.188$ at 1 month and $7.50 \pm 2.675$ at 3 months. In Phaco patients, it was $7.538 \pm 1.906$ at 1 week, $6.718 \pm 1.723$ at 1 month, and $7.513 \pm 1.959$ at 3 months.

We saw that the mean and standard deviation of tear meniscus height at 1 week was $0.25 \pm 0.09$ in SICS patients, at 1 month was $0.20 \pm 0.116$, at 3 months $0.25 \pm 0.09$. Whereas in Phaco patients, tear meniscus height was found out to be $0.333 \pm 0.0692$ at 1 week, $0.3 \pm 0.075$ at 1 month, $0.333 \pm 0.069$ at 3 months.

Likewise the mean and standard deviation of schirmer's test at 1 week was 7.30 2.246 in SICS patients, at 1 month was $6.60 \pm 1.754$ and at 3 months was $7.83 \pm 2.036$. Whereas in Phaco patients, schirmer's test was found out to be $7.615 \pm 1.862$ at 1 week, $6.949 \pm 1.663$ at 1 month, $7.846 \pm 1.981$ at 3 months. The preoperative and postoperative findings are shown in table 1 to 4 .

TABLE 1: PREOPERATVE VALUES OF DIFFERENT DRY EYE INDICES FOR PATIENTS UNDERGOING CATARACT SURGERY (n=69)

\begin{tabular}{|l|c|c|c|c|c|}
\hline & $\begin{array}{c}\text { TEAR } \\
\text { MENISCUS } \\
\text { HEIGHT (TMH) } \\
\text { (Mean } \pm \text { SD) }\end{array}$ & $\begin{array}{c}\text { SCHIRMER'S } \\
\text { TEST (ST) } \\
(\text { Mean } \pm \text { SD) }\end{array}$ & $\begin{array}{c}\text { TEAR BREAK UP } \\
\text { TIME (TBUT) } \\
\text { (Mean } \pm \text { SD) }\end{array}$ & $\begin{array}{c}\text { OSDI } \\
\text { (Mean } \pm \text { SD) }\end{array}$ & $\begin{array}{c}\text { IMPRESSION } \\
\text { CYTOLOGY } \\
(\text { Mean } \pm \text { SD) }\end{array}$ \\
\hline SICS & $0.55 \pm 0.166$ & $9.23 \pm 2.112$ & $9.48 \pm 3.212$ & $56.18 \pm 10.926$ & $208.80 \pm 59.120$ \\
\hline PHACO & $0.554 \pm 0.170$ & $9.231 \pm 2.117$ & $9.487 \pm 2.489$ & $54.482 \pm 9.001$ & $237.937 \pm 238.100$ \\
\hline
\end{tabular}

TABLE 2: VALUES OF DIFFERENT DRY EYE INDICES FOR PATIENTS UNDERGOING CATARACT SURGERY AFTER 1 WEEK OF SURGERY (n=69)

\begin{tabular}{|l|c|c|c|c|}
\hline & $\begin{array}{c}\text { TEAR MENISCUS } \\
\text { HEIGHT (Mean } \pm \\
\text { SD) }\end{array}$ & $\begin{array}{c}\text { SCHIRMER'S TEST } \\
(\text { Mean } \pm \text { SD) }\end{array}$ & $\begin{array}{c}\text { TEAR BREAK UP TIME } \\
(\text { Mean } \pm \text { SD) }\end{array}$ & $\begin{array}{c}\text { OSDI } \\
(\text { Mean } \pm \text { SD) }\end{array}$ \\
\hline SICS & $0.25 \pm 0.09$ & $7.30 \pm 2.246$ & $7.57 \pm 2.285$ & $50.45 \pm 10.247$ \\
\hline PHACO & $0.333 \pm 0.0692$ & $7.615 \pm 1.862$ & $7.538 \pm 1.906$ & $47.360 \pm 7.525$ \\
\hline
\end{tabular}

TABLE 3: VALUES OF DIFFERENT DRY EYE INDICES FOR PATIENTS UNDERGOING CATARACT SURGERY AFTER 1 MONTH OF SURGERY (n=69)

\begin{tabular}{|l|c|c|c|c|}
\hline & $\begin{array}{c}\text { TEAR MENISCUS } \\
\text { HEIGHT } \\
(\text { Mean } \pm \text { SD) }\end{array}$ & $\begin{array}{c}\text { SCHIRMER'S TEST } \\
(\text { Mean } \pm \text { SD) }\end{array}$ & $\begin{array}{c}\text { TEAR BREAK UP } \\
\text { TIME } \\
(\text { Mean } \pm \text { SD) }\end{array}$ & $\begin{array}{c}\text { OSDI } \\
(\text { Mean } \pm \text { SD) }\end{array}$ \\
\hline SICS & $0.20 \pm 0.116$ & $6.60 \pm 1.754$ & $6.80 \pm 2.188$ & $39.86 \pm 9.215$ \\
\hline PHACO & $0.300 \pm 0.075$ & $6.949 \pm 1.663$ & $6.718 \pm 1.723$ & $40.628 \pm 8.907$ \\
\hline
\end{tabular}

TABLE 4: VALUES OF DIFFERENT DRY EYE INDICES FOR PATIENTS UNDERGOING CATARACT SURGERY AFTER 3 MONTH OF SURGERY $(n=69)$

\begin{tabular}{|c|c|c|c|c|c|}
\hline & $\begin{array}{c}\text { TEAR } \\
\text { MENISCUS } \\
\text { HEIGHT } \\
\text { (Mean } \pm \text { SD) }\end{array}$ & $\begin{array}{c}\text { SCHIRMER'S } \\
\text { TEST } \\
(\text { Mean } \pm \text { SD) }\end{array}$ & $\begin{array}{c}\text { TEAR BREAK } \\
\text { UP TIME } \\
(\text { Mean } \pm \text { SD })\end{array}$ & $\begin{array}{c}\text { OSDI } \\
(\text { Mean } \pm \text { SD) }\end{array}$ & $\begin{array}{c}\text { IMPRESSION } \\
\text { CYTOLOGY } \\
\text { (Mean } \pm \text { SD) }\end{array}$ \\
\hline SICS & $0.25 \pm 0.09$ & $7.83 \pm 2.036$ & $7.50 \pm 2.675$ & $37.76 \pm 9.607$ & $143.16 \pm 63.043$ \\
\hline PHACO & $0.333 \pm 0.069$ & $7.846 \pm 1.981$ & $7.513 \pm 1.959$ & $38.051 \pm 8.428$ & $184.436 \pm 59.904$ \\
\hline
\end{tabular}




\section{Discussion}

Dry eye disease is a multifactorial disease of the tear film and ocular surface that results in symptoms of discomfort, visual disturbance, and tear instability with potential damage to the ocular surface. It is accompanied by increased osmolarity of the tear film and inflammation of the ocular surface, as described in the International Dry Eye Work Shop, $2007^{8}$.

Dry eye produces discomfort and reduced vision when the tear film becomes chronically unstable and repeatedly breaks up into dry spots between blinks, exposing the corneal and conjunctival epithelium to evaporation (Parson, et al., $2007^{9}$ ).

Cataract surgery is the most successful and classic surgery, which has given good visual acuity to many patients. Cataract operations have substantially increased from 16 lakh in 1992-93 to 59.1 lakh in 2009-10 according to the annual report 2010-11 of National Programme for Control of Blindness. ${ }^{10}$

The etiology of dry eye following cataract surgery is characterized by presence of either pre-existing dry eye or surgically-induced dry eye. Various factors might affect the ocular surface environment after cataract surgery. Most important is corneal desensitization ${ }^{5}$. Superficial punctate keratitis, recurrent filamentary keratitis, secondary infections including conjunctivitis, infective keratitis, persistent or recurrent epithelial defects, stromal keratolysis and corneal ulceration have been reported in dry eye patients after cataract surgery notably conventional extracapsular cataract extraction (ECCE) by Ram et al in $2002^{5}$.

The present study was done on sixty nine patients attending the outpatient department having cataract and were scheduled for cataract operation. Amongst them, 30 patients were scheduled for small incision cataract surgery (SICS) and 39 patients were scheduled for Phacoemulsification (Phaco).

$\mathrm{Li}$, et al in 2007 had done a study to investigate dry eye disease and analyse the pathogenic factors in patients after cataract surgery ${ }^{3}$. They had taken age related cataract patients who were scheduled for phacoemulsification, and led to the conclusion that dry eye can develop or deteriorate after cataract surgery if not treated on time. Dry eye appeared at 1 week postoperatively and peaked at 1 month. Similar findings were observed by Liu, et al., ${ }^{11}$ Cho, et al., ${ }^{2}$ and Gharaee, et al. ${ }^{12}$

Majority (89\%) of our patients had no fluorescein staining at the onset of our study. The staining reached a peak at 1 month (32\%) and gradually decreased by the end of 3 months (15\%) so that at the end of our study, $14.4 \%$ of the patients had moderate grade stain while the rest had no stain. This is similar to the studies conducted by Ram, et al., ${ }^{5}$ and $\mathrm{Li}$, et al., ${ }^{3}$. However Liu, et al found out that fluorescein staining got increased in the $1^{\text {st }}$ week follow-up visits, and decreased subsequently in the next visits. ${ }^{11}$

The TMH values of both the cases and control group were found to be low at the beginning of the study preoperatively. The TMH values were lowest at 1 month, while it showed slight improvement at 3 months. The lowest value of TBUT was seen at 1 month. Cho, et al., in their study found out that TMH value decreased in all the follow up visits from the preoperative value in the non-dry eye group. ${ }^{2}$ Gharaee, et al., also showed a decreased TMH value at 3 months follow up visit. ${ }^{12}$ Whereas Sitompul, et al., found out that there was no significant difference in the TMH values in the follow-up visits in both SICS and phaco groups. ${ }^{13}$

The OSDI score steadily decreased in the follow up period upto 3 months although some of the dry eye tests improved like TMH, fluorescein staining after 1 month. This indicates that the patients continued to have symptoms of dry eye although there tear functions improved. This was seen in other studies also and is probably due to the improvement in visual acuity on one hand and ocular discomfort and environmental triggers noted on the other hand after cataract surgery. Li, et al., did not find any statistically significant change of overall OSDI score before and after cataract surgery. ${ }^{3}$ Sitompul, et al., found out that OSDI score decreased on day 14 in patients who had undergone SICS, but the overall OSDI score improved after phaco in the follow up visits. ${ }^{13}$

Conjunctival impression cytology was done preoperatively and at 3 months postoperatively. In the control group the mean of the number of goblet cells found in impression cytology was 214.92 and the standard deviation was 54.59 preoperatively. At the end of 3 months, the mean number of goblet cells did not show much change. But, in the study group, the number of goblet cells decreased significantly from $223.37 \pm 148.61$ to $163.79 \pm 61.47$ at 3 months. Furthermore, squamous metaplasia was noted in some of the impression cytology specimens after cataract surgery, at 3 months follow-up. Li, et al., also recorded decrease in goblet cells and squamous metaplasia in their study. They had obtained the samples from explosive region, upper lid and lower lid covered region while we have obtained samples from upper lid covered region only. ${ }^{3}$ 
Dogru et al in 2001 reported that TBUT and ST-I scores significantly decreased in non-insulin-dependent diabetic mellitus ${ }^{14}$. Patients with diabetes presented with cataract commonly. And neurological and biochemical changes due to diabetes affect innervations and secretory function of lacrimal gland.

\section{Comparative analysis of dry eye parameters between the two surgical groups:}

We analysed the patients according to those who had undergone manual small incision cataract surgery and those who had undergone phacoemulsification, and found out that pre-operatively the mean and standard deviation of tear meniscus height, schirmer's test, tear film breakup time, ocular surface disease index and impression cytology were respectively $0.55 \pm 0.166,9.23 \pm 2.112,9.48 \pm 3.212,56.18 \pm 10.926$ and $208.80 \pm 59.120$ in patients who underwent SICS and 0.55 $\pm 0.170,9.231 \pm 2.117,9.487 \pm 2.489,54.482 \pm 9.001$ and $237.937 \pm 238.1$ in patients who underwent Phacoemulsification. Thus the two groups were similar in their dry eye status.

After SICS and Phaco surgery all the dry eye tests (TMH, TBUT, ST-I and fluorescein staining) showed a lower value indicating that the tear production of the eye was affected. The values were lowest at 1 month after which they improved. The OSDI score also showed a decline and incidentally it did not show any improvement after the first month. Several studies have reported OSDI score does not correlate with the traditional objective clinical measures of dry eye ${ }^{3,13}$. This may be due to lack of sensitivity of these measures to capture the complete range of ocular surface and tear abnormality that produces the typical dry eye symptoms.

S.Khanal, et al., ${ }^{15}$ also reported a similar finding in their study. The probable cause is thought to be due to a decrease in tear production and increase in tear evaporation. The decrease in tear production is due to decrease in corneal sensitivity while use of steroid drops and other drops containing benzalkonium chloride used post operatively destabilizes the lipid layer resulting in increased evaporation ${ }^{15}$. They also discussed that the loss of corneal sensitivity after cataract surgery often persisted for more than two years and can be permanent. There is deficiency of aqueous layer and an unstable tear film leading to superficial punctate keratopathy. We too observed increase in fluorescein staining following cataract surgery up to a period of one month after which it decreased.

\section{Conclusion}

All the dry eye tests conducted on the eyes undergoing cataract surgery showed deterioration following surgery. The mean values of TMH, ST-I and TBUT were below normal at 1 week, 1 month and 3 months follow up. The lowest value was recorded at 1 month. Thus cataract surgery affected both tear quantity and tear quality. The fluorescein staining of the cornea was of mild to moderate grade in $75 \%$ of cases while $13.04 \%$ had severe grade at the end of 3 months. Thus after cataract surgery the corneal epithelium remained unstable due to the altered corneal sensation and tear dynamics. The OSDI score steadily decreased in the follow up period although some of the dry eye tests improved marginally. This indicates that the patients continued to have symptoms of dry eye although his tear functions improved. The symptoms of dry eye disease may not corroborate with the actual dry eye status. After cataract surgery the impression cytology of conjunctiva showed significant goblet cell loss $(\mathrm{p}<0.001)$ and squamous metaplasia was seen in some of the slides. This indicates that surgery causes alteration in tear composition resulting in unstable tear film. Both SICS and Phaco surgery caused significant decrease in TMH, TBUT and ST-I values at 1 week, 1 month and 3 months. Fluorescein staining, OSDI score and impression cytology also showed deterioration in the follow up period. Although the two surgeries differ in terms of procedure and technology but both caused similar dry eye condition as measured by various tests that we have conducted in our study. The medications used in the preoperative and postoperative period were common to both the groups. Some of these topical eye drops contained preservatives. The steroid drops and the other eye drops containing preservatives like benzalkonium chloride are known to cause tear film instability. This may have contributed to the dry eye condition post operatively. Thus we conclude that cataract surgery can cause or aggravate dry eye and affect the dry eye test values in the post operative period upto 3 months. This holds true for both SICS and Phaco surgery. But before surgery, patients must be informed about the possible aggravation of dry eye symptoms and artificial tears should be prescribed for attenuating corneal damage and dry eye symptoms.

\section{References}

[1]. Srinivasan, R, Agarwal V, Suchismitha T, and Kavitha S. Dry eye after Phacoemulsification. AIOC Proceedings. 2008.

[2]. Cho YK, Man Soo Kim. Dry Eye After Cataract Surgery and Associated Intraoperative Risk Factors. Korean J Ophthalmol. 2009 June; 23(2): 65-73.

[3]. Li XM, Hu L, Hu J, and Wang W. Investigation of Dry Eye Disease and Analysis of the Pathogenic Factors in Patients after Cataract Surgery. Cornea. 2007; 26(Suppl. 1): S16-S20.

[4]. Ram J, Sharma A, Pandav SS, Gupta A, Bambery P. Cataract surgery in patients with dry eyes. J. Cataract Refract Surg. 1998 Aug; 24(8): 1119-24. 
[5]. Ram J, Gupta A, Brar GS, Kaushik S, Gupta A. Outcomes of phacoemulsification in patients with dry eye. J Cataract Refract Surg. 2002 March; 28: 1386-1389.

[6]. Bourne RR, Minassian DC, Dart JK, Rosen P, Kaushal S, Wingate N. Effect of cataract surgery on the corneal endothelium: modern phacoemulsification compared with extracapsular cataract surgery. Ophthalmol. 2004; 111: 679-685.

[7]. Ocular Surface Disease Index. Data on file, Allergan, Inc. 1995.

[8]. The definition and classification of dry eye disease: report of the definition and classification subcommittee of the International Dry Eye WorkShop (2007). The Ocul Surf. 2007; 5(2): 75-92.

[9]. Sihota, R, and Tandon R. Parsons' Disease of the Eye, $20^{\text {th }}$ ed., New Delhi: Elsevier, 2007.

[10]. Ministry of Health and Family Welfare, Govt. Of India. National Programme for Control of Blindness, Annual Report 2010-11, MOHFW, Govt of India, p. 109-111.

[11]. Liu Xi, Yang-shun Gu, Ye-sheng Xu. Changes of tear film and tear secretion after phacoemulsification in diabetic patients. $\mathbf{J}$ Zhejiang Univ Sci B. 2008 April; 9(4): 324-328.

[12]. Gharaee H, Mousavi MN, Daneshvar R, Hosseini M, Sazande S. Effect of clear corneal incision location on tear film following phacoemulsification surgery. Iranian Journal of Ophthalmology. 2009; 21(3): 29-34.

[13]. Sitompul R, Sancoyo GS, Hutauruk JA, Gondhowiardjo TD. Sensitivity Change in Cornea and Tear layer due to Incision Difference on Cataract Surgery with Either Manual Small-Incision Cataract Surgery or Phacoemulsification. Cornea. 2008 Sep; 27(Suppl. 1): S13-S18.

[14]. Dogru M, Katakami C, Inoue M. Tear function and ocular surface changes in Non-Insulin Dependent Diabetes Mellitus. Ophthalmology. 2001; 108(3): 586-92.

[15]. Khanal S, Tomlinson A, Esakowitz L, Bhatt P, Jones D, Nabili S, Mukerji S. Changes in corneal sensitivity and tear physiology after phacoemulsification. Opthal. Phsiol. Opt. 2008; 28: 127-134. 